\title{
Temporal cleaning of a high energy fiber-based ultrafast laser using cross-polarized wave generation
}

\author{
Yoann Zaouter, ${ }^{1 *}$ Lourdes Patricia Ramirez, ${ }^{2}$ Dimitrios N. Papadopoulos, ${ }^{3}$ Clemens Hönninger, ${ }^{1}$ \\ Marc Hanna, ${ }^{2}$ Frédéric Druon, ${ }^{2}$ Eric Mottay ${ }^{1}$, Patrick Georges ${ }^{2}$ \\ ${ }^{I}$ Amplitude Systèmes, 6 allée du Doyen Georges Brus, 33600 Pessac, France \\ ${ }^{2}$ Laboratoire Charles Fabry de l'Institut d'Optique, CNRS, Université Paris Sud, \\ RD 128, 91127 Palaiseau Cedex, France \\ ${ }^{3}$ Institut de la Lumière Extrême, CNRS, Ecole Polytechnique, ENSTA Paristech, \\ Institut d'Optique, Université Paris Sud, Palaiseau Cedex, France \\ *Corresponding author: yzaouter@amplitude-systemes.fr
}

Received Month X, XXXX; revised Month X, XXXX; accepted Month X, XXXX; posted Month X, XXXX (Doc. ID XXXXX); published Month X, XXXX

\begin{abstract}
We report the use of cross-polarized wave generation to perform both pulse shortening and temporal cleaning of a highenergy ytterbium-doped fiber-based femtosecond laser system. The nonlinear processes allow both highly efficient nonlinear conversion of $20 \%$ and a large compression ratio of 3.5, with an inherently improved coherent and incoherent contrast. This results in the generation of $37 \mu \mathrm{J}, 115 \mathrm{fs}$ pulses at a repetition rate of $100 \mathrm{kHz}$ with high temporal quality. (C) 2011 Optical Society of America
\end{abstract}

OCIS Codes: $320.7110,190.4360,140.3510$

High optical temporal contrast ratio is an essential property for ultrashort laser sources that are used in highfield physics experiments. The cross-polarized wave generation effect (XPW), with only one demonstration at the wavelength of $1 \mu \mathrm{m}$ [3], has been successfully used to enhance this contrast, mostly for Ti:Sapphire based ultrafast laser sources [1-2], The main limitation of these laser sources is that thermal effects and the complexity of the required pump systems limit the repetition rate, or equivalently the average power available, to a few tens of Watts for the most advanced systems.

At the other end of the spectrum of ultrafast laser sources, ytterbium-doped fiber-based systems benefit from their guiding geometry to offer very high average power, of the order of $1 \mathrm{~kW}$ [4], and excellent beam quality, in relatively simple systems. The available pulse energies have immensely progressed during last decade to reach levels that are compatible with high-field physics experiments such as high-harmonic generation [5], but remain limited by the geometry of the amplifying medium. Aside from limited pulse energy, high-energy fiber-based sources suffer from longer pulsewidths, on the order of $400 \mathrm{fs}$, because of the limited gain bandwidth of ytterbium in the glass matrix. Nonlinear amplification techniques can be used to obtain moderately energetic pulses with shorter durations [6], but in these setups, residual higher-order spectral phase degrades the pulse quality and the coherent temporal contrast.

In this work, we propose to use the XPW technique at the output of a femtosecond fiber chirped-pulse amplifier system delivering $200 \mu \mathrm{J}, 405 \mathrm{fs}$ pulses at a wavelength of $1.03 \mu \mathrm{m}$ and a repetition rate of $100 \mathrm{kHz}$ to obtain shorter pulses that feature a very good temporal quality and enhanced coherent and incoherent contrast. In order to obtain the shortest possible pulses from the nonlinear XPW stage, a controlled amount of self- and cross-phase modulation (SPM, XPM) can be allowed in the same crystal [2]. This permits us to obtain high contrast, $37 \mu \mathrm{J}$ pulses compressed down to 115 fs duration at a repetition rate of $100 \mathrm{kHz}$, corresponding to an average power of 3.7 W. The obtained temporal profile shows an excellent quality, with a temporal Strehl ratio of $93 \%$. We believe that this experimental demonstration of the efficiency of XPW on fiber-based femtosecond systems constitutes a significant step towards high contrast high repetition rate femtosecond laser systems.

Our experimental setup is depicted in Fig. 1. The fiber amplifier used to seed the XPW experiment is based on the well known fiber chirped-pulse amplification (FCPA) scheme. Low energy ultrashort pulses generated by a mode-locked oscillator are stretched in time prior to amplification in order to minimize the amount of accumulated non-linear phase shifts during amplification. An acousto-optic modulator is mounted upstream the amplifier to reduce the repetition rate of the oscillator pulse train down to $100 \mathrm{kHz}$. The amplifier is made of an $80 \mathrm{~cm}$-long rod type photonic crystal fiber used in double pass configuration. This fiber possesses a very large core diameter of $80 \mu \mathrm{m}$, allowing for a significant non-linearity reduction in comparison to standard telecom fibers, together with a high numerical aperture air-clad diameter of $200 \mu \mathrm{m}$ that ensures high pump absorption of up to 27 $\mathrm{dB} / \mathrm{m}$. The fiber is pumped by a $90 \mathrm{~W}$ diode fiber coupled to a $400 \mu \mathrm{m}$ diameter fiber with a numerical aperture of 0.22 . After amplification, the high energy pulses are optically isolated and compressed in a transmission grating based compressor.

After the FCPA, the obtained pulses are sent to the nonlinear temporal filter stage. It is composed of a nonlinear crystal placed between crossed polarizers and two lenses with focal length $200 \mathrm{~mm}$. The polarizer pair extinction ratio is greater than $10^{4}$. The nonlinear crystal 
is a $4 \mathrm{~mm}$-long holographic-cut barium fluoride $\mathrm{BaF}_{2}$ that has been shown to exhibit a good efficiency and reduced dependence of the optimal angle to the input intensity [2] . The crystal position is adjusted after the focal plane to optimize the XPW efficiency while avoiding optical damage that arises at intensities around $10^{13} \mathrm{~W} / \mathrm{cm}^{2}$. At the output of this nonlinear stage, a tunable dispersion line using a single $1250 \mathrm{l} / \mathrm{mm}$ grating compressor is used to compress the pulses. The efficiency of this compressor is $92.5 \%$.

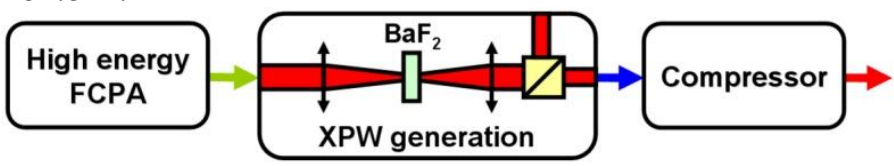

Fig. 1. (Color online) Experimental setup.

The output energy of the temporal cleaning stage (before compression) and the corresponding XPW conversion efficiency is shown in Fig. 2(a) as a function of input energy. For input energies below $100 \mu \mathrm{J}$, the nonsaturated regime approximation holds and results in a cubic dependence of the output power, or quadratic dependence of the efficiency. For higher input intensities, saturation effects and dephasing of the pump and XPW signal cause a saturation of the energy transfer. The maximum overall measured efficiency is $20.5 \%$ at $200 \mu \mathrm{J}$. Further scaling of the intensity in the crystal results in white light generation and optical damage. Taking into account the losses at uncoated facets of the $\mathrm{BaF}_{2}$ crystal and optical components, this corresponds to an internal XPW efficiency of $25 \%$, a rather high value for a single crystal setup. Note that the maximal theoretical efficiency assuming a perfect Gaussian beam is, $52 \%$ but can not be practically reached because it requires intensities well above the bulk damage threshold. Figure 2(b) shows the spectra at maximum power at the output of the FCPA and XPW stage. The XPW spectrum exhibits a significant broadening of almost a factor of 2, to reach $12 \mathrm{~nm}$ FWHM, and a reshaping toward a more Gaussian-like shape typical from the XPW process. Therefore, temporal shortening can be expected.
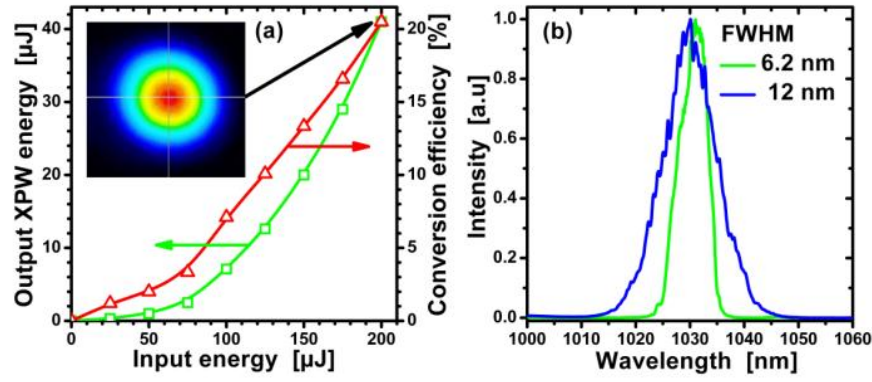

Fig. 2. (Color online) (a) Output XPW energy (green open square) and XPW efficiency (red open triangle) as a function of input energy together with output beam profile at the highest energy obtained (inset). (b) Output spectra (green) before and (blue) .after XPW stage.

The temporal characteristics of the pulses at various locations in the system are shown in Fig. 3. The experimental data is obtained using a scanning autocorrelator and a single-shot second-harmonic generation frequency-resolved optical gating (FROG) setup. Redundancy of the measurements ensures a thorough characterization.

The $200 \mu \mathrm{J}$ pulses at the output of the amplifier system (green curves in Fig. 3) exhibit a duration of $405 \mathrm{fs}$ and FWHM spectral width of $6.4 \mathrm{~nm}$, corresponding to a time bandwidth product of 0.74 . This value can be explained by the accumulated nonlinear phase in the fiber amplifier that is partially but not fully compensated by a mismatch between the FCPA stretcher and compressor. This residual higher-order spectral phase is translated in the temporal domain to a low pedestal that extends over $2 \mathrm{ps,}$ an example of non perfect coherent contrast.

The XPW process is known to remove spectral phase distortions due to the highly selective temporal filtering. After the XPW stage the pulses have a duration of $340 \mathrm{fs}$, and the pedestal has completely disappeared. The reduction by a factor of 1.2 of the duration is smaller than the expected value of $\sqrt{3}$ for the small signal regime because of the saturation of the conversion due to pump depletion. In this high efficiency regime, the effects of SPM and XPM can not be neglected and partially impart an additional broadening of the spectrum. The spectral phase induced by these effects on the XPW pulses can be clearly identified on the retrieved FROG at the output of the XPW stage.
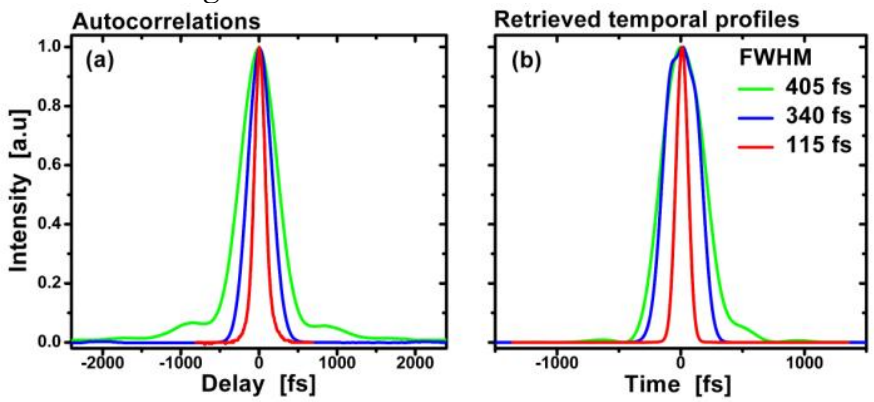

Fig. 3. (Color online) (a) Autocorrelations and (b) retrieved temporal profiles from the SHG FROG measurements; taken at the output of (green) FCPA at $200 \mu \mathrm{J}$, (blue) XPW stage at $41 \mu \mathrm{J}$ and (red) after compression at $37 \mu \mathrm{J}$. All FROG retrieval errors are less than $5.10-{ }^{4}$ on $256 \times 256$ grids.

We therefore set a $1250 \mathrm{l} / \mathrm{mm}$ transmission grating-based compressor in order to compensate this remaining phase. The simple-pass geometry allows us to improve the overall compression efficiency to $92.5 \%$ at the expense of some negligible spatial chirp. Indeed, optimal compression of the XPW beam is obtained for an equivalent distance between the gratings of only $0.8 \mathrm{~mm}$. In such a case, the entire spectrum is spatially spread over $30 \mu \mathrm{m}$. Compared to a beam diameter of $2 \mathrm{~mm}$ the spatial chirp can be considered insignificant. The compressed pulsewidth is $115 \mathrm{fs}$, with a time bandwidth product of 0.42 . This corresponds to a significant pulse compression factor of $3.5,35 \%$ higher than the previously best obtained by an $\mathrm{XPW}$ stage at $800 \mathrm{~nm}$ [7]. This higher compression ratio can be explained by a reduced sensitivity to dispersion effects for the longer pulses at $1 \mu \mathrm{m}$ in the $4 \mathrm{~mm}$-long BaF2. Moreover the pulse temporal quality is almost perfect due to the temporal cleaning of the XPW. The XPW peak power transmission, defined as the ratio between the peak power of the compressed XPW pulses and the FCPA pulses, is 0.65 , a record value, owing to the additional spectral broadening by XPM and SPM. The 
final output pulses exhibit a peak power of 320 MW with clean temporal profile and enhanced contrast at a repetition rate of $100 \mathrm{kHz}$ and an average power of $3.7 \mathrm{~W}$. Simulations based on the coupled nonlinear propagation equations that govern XPW generation were performed to ensure our understanding of the process. We used the model described in [8] that takes into account third order nonlinear processes, and added dispersion up to third order. The input to the propagation algorithm is the FROG-retrieved electric field at the output of the amplifier. The results are displayed on Fig. 4, and show excellent agreements with the measured data. The computed XPW efficiency in this case is $26 \%$, and corresponds to a maximum optical intensity of $2.7 \times 10^{12}$ $\mathrm{W} / \mathrm{cm}^{2}$, close to the damage threshold. These values fit very well with the experimental parameters, and confirm our global understanding of the nonlinear mechanisms. Compared with traditional SPM only-based pulse compression methods, such as propagation in a gas-filled capillary, XPW brings an additional temporal filtering that results in a much better output pulse temporal quality and contrast, or equivalently much smoother snectral nhase at the exnense of rediced efficiency.

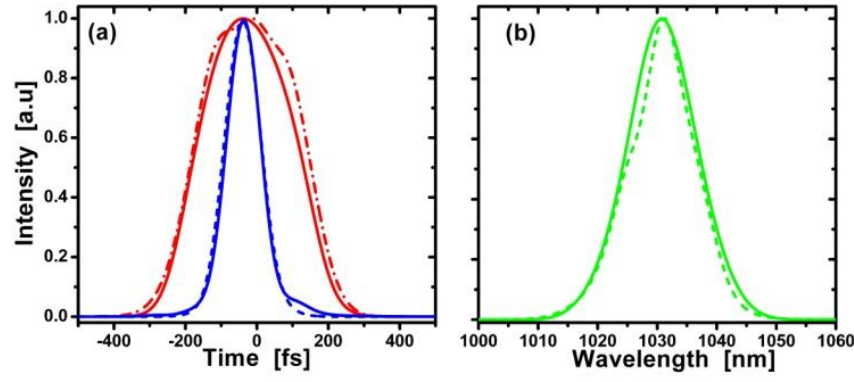

Fig. 4. (Color online) (a) Simulated temporal profiles of XPW pulses (solid red) before and (solid blue) after compression. Experimental temporal profiles retrieved by FROG for the XPW pulse (dash dot red)before and (dash blue) after compression. (b) Spectrum of (solid green) the simulated and (dash green) retrieved spectrum by FROG of the XPW pulses.

We now discuss spatial aspects of the experiment. The XPW process also takes place in the spatial domain, leading to an output profile smaller than the input profile. An interesting aspect of the XPW process at the output of high average power fiber amplifiers is that, if higher-order transverse modes are present in the output beam, the corresponding pulses in time arrive at the XPW crystal with a group-velocity delay, and due to the highly nonlinear behavior of XPW, are filtered out. In our experiment, the beam profile of the XPW beam, shown in inset of Figure 2(a), exhibits a Gaussian profile similar to the output beam of the FCPA despite being smaller in size. Since the FROG traces are acquired using the whole beam the low FROG retrieval errors indicate that spatiotemporal distortions are low.

In conclusion, we demonstrated, for the first time to the best of our knowledge, the temporal filtering of a high energy fiber chirped pulse amplifier by means of crosspolarized wave generation. Assisted by the onset of SPM and XPM, the XPW process not only removes the pedestal of the original pulses but also allows for significant pulse shortening, with a compression ratio of 3.5. Furthermore, with an average power of $4.1 \mathrm{~W}$, in the single crystal configuration, we report the highest average power generated through XPW process. No average powerrelated crystal degradation was observed during the experiment. The peak power after filtering and compression of the XPW pulses is $320 \mathrm{MW}(37 \mu \mathrm{J}$ and 115 fs) corresponding to a peak power transmission of 0.65 . The authors believe that this source could be integrated in a fiber-based double CPA architecture towards the multiGW regime, or be used as a seeder for a large scale laser chain where a good coherent and incoherent contrast is critical. Future works will focus on further pulse shortening based on a nonlinear fiber amplifier configuration and the remarkable spectral cleaning effect of XPW previously demonstrated at $800 \mathrm{~nm}[9,10]$, as well as efficiency improvement based on a double crystal architecture [11].

The authors acknowledge A. Jullien and R. LopezMartens for fruitful discussions. Dimitrios N. Papadopoulos acknowledges the financial support from the ILE 07-CPER 017-01 contract.

\section{References}

1. A. Jullien, O. Albert, F. Burgy, G. Hamoniaux, J.-P. Rousseau, J.-P. Chambaret, F. Augé-Rochereau, G. Chériaux, J. Etchepare, N. Minkovski, S. M. Saltiel, Optics Letters 30, 920-922 (2005).

2. A. Jullien, J.-P. Rousseau, B. Mercier, L. Antonucci, O. Albert, G. Chériaux, S. Kourtev, N. Minkovski, S. M. Saltiel, Optics Letters 33, 2353--2355 (2008).

3. A Cotel, A Jullien, N. Forget, O. Albert, G. Chériaux, C. Le Blanc, Applied Physics B 83, 7-10 (2006).

4. T. Eidam, S. Hanf, E. Seise, T. V. Andersen, T. Gabler, C. Wirth, T. Schreiber, J. Limpert, A. Tünnermann, Optics Letters 35, 94-96 (2010).

5. J. Boullet, Y. Zaouter, J. Limpert, S. Petit, Y. Mairesse, B. Fabre, J. Higuet, E. Mével, E. Constant, and E. Cormier, Optics Letters 34, 1489-1491 (2009).

6. D. N. Papadopoulos, M. Hanna, F. Druon, and P. Georges, IEEE Journal of Selected Topics in Quantum Electronics 15, 182-186 (2009).

7. L. P. Ramirez, D. N. Papadopoulos, A. Pellegrina, P. Georges, F. Druon, P. Monot, A. Ricci, A. Jullien, X. Chen, J. P. Rousseau, and R. Lopez-Martens, Optics Express 19, 93-98 (2011).

8. L. Canova, S. Kourtev, N. Minkovski, A. Jullien, R. Lopez-Martens, O. Albert, S. M. Saltiel, Applied Physics Letters 92, 231102-1 (2008).

9. Y. Zaouter, D. N. Papadopoulos, M. Hanna, J. Boullet, L. Huang, C. Aguergaray, F. Druon, E. Mottay, P. Georges, and E. Cormier, Optics Letters 33, 107-109 (2008).

10. A. Jullien, X. Chen, A. Ricci, J.-P. Rousseau, R. LopezMartens, L. P. Ramirez, D. Papadopoulos, A. Pellegrina, F. Druon and P. Georges, Applied Physics B Online First, 15 September 2010.

11. A. Jullien, O. Albert, G. Chériaux, J. Etchepare, S. Kourtev, N. Minkovski, and S. M. Saltiel, Optics Express 14, 2760-2769 (2006). 
References

[1] A. Jullien, O. Albert, F. Burgy, G. Hamoniaux, J.-P. Rousseau, J.-P. Chambaret, F. Augé-Rochereau, G. Chériaux, J. Etchepare, N. Minkovski, S. M. Saltiel, " $10^{\wedge}-10$ temporal contrast for femtosecond ultraintense lasers by cross-polarized wave generation," Optics Letters 30, 920-922 (2005).

[2] A. Jullien, J.-P. Rousseau, B. Mercier, L. Antonucci, O. Albert, G. Chériaux, S. Kourtev, N. Minkovski, S. M. Saltiel, "Highly efficient nonlinear filter for femtosecond pulse contrast enhancement and pulse shortening,” Optics Letters 33, 2353--2355 (2008).

[3] A Cotel, A Jullien, N. Forget, O. Albert, G. Chériaux, C. Le Blanc, "Nonlinear temporal pulse cleaning of a 1- $\mu$ m optical parametric chirped-pulse amplification system,” Applied Physics B 83, 7-10 (2006).

[4] T. Eidam, S. Hanf, E. Seise, T. V. Andersen, T. Gabler, C. Wirth, T. Schreiber, J. Limpert, A. Tünnermann, ’Femtosecond fiber CPA system emitting $830 \mathrm{~W}$ average output power," Optics Letters 35, 94-96 (2010).

[5] J. Boullet, Y. Zaouter, J. Limpert, S. Petit, Y. Mairesse, B. Fabre, J. Higuet, E. Mével, E. Constant, and E. Cormier, "High-order harmonic generation at a megahertz-level repetition rate directly driven by an ytterbium-doped-fiber chirped-pulse amplification system," Optics Letters 34, 1489-1491 (2009).

[6] D. N. Papadopoulos, M. Hanna, F. Druon, and P. Georges, "Compensation of gain narrowing by self-phase modulation in high-energy ultrafast fiber chirped-pulse amplifiers," IEEE Journal of Selected Topics in Quantum Electronics 15, 182-186 (2009).

[7] L. P. Ramirez, D. N. Papadopoulos, A. Pellegrina, P. Georges, F. Druon, P. Monot, A. Ricci, A. Jullien, X. Chen, J. P. Rousseau, and R. Lopez-Martens, "Efficient cross polarized wave generation for compact, energy-scalable, ultrashort laser sources," Optics Express 19, 93-98 (2011)

[8] L. Canova, S. Kourtev, N. Minkovski, A. Jullien, R. Lopez-Martens, O. Albert, S. M. Saltiel, "Efficient generation of cross-polarized femtosecond pulses in cubic crystals with holographic cut orientation," Applied Physics Letters 92, 231102-1 (2008).

[9] Y. Zaouter, D. N. Papadopoulos, M. Hanna, J. Boullet, L. Huang, C. Aguergaray, F. Druon, E. Mottay, P. Georges, and E. Cormier, "Stretcher-free high energy nonlinear amplification of femtosecond pulses in rod-type fibers," Optics Letters 33, 107-109 (2008).

[10] A. Jullien, X. Chen, A. Ricci, J.-P. Rousseau, R. Lopez-Martens, L. P. Ramirez, D. Papadopoulos, A. Pellegrina, F. Druon and P. Georges, "High-fidelity front-end for high-power, high temporal quality few-cycle lasers," Applied Physics B Online First, 15 September 2010 .

[11] A. Jullien, O. Albert, G. Chériaux, J. Etchepare, S. Kourtev, N. Minkovski, and S. M. Saltiel, "A two crystal arrangement to fight efficiency saturation in cross-polarized wave generation," Optics Express 14, 2760-2769 (2006) 